\title{
Gestión del riesgo crediticio y el índice de morosidad en colaborares de una entidad financiera del departamento de Puno
}

Credit risk management and delinquency rate in collaborators of a financial institution in the department of Puno

\section{Carmen Rosa Vilca Mamani a(D)*, Julio Samuel Torres-MirandabiD \\ a,b Universidad Peruana Unión, Juliaca, Perú}

\section{Resumen}

En los últimos tiempos la gestión de riesgo crediticio y el índice de morosidad es causa de mucha preocupación para las empresas dedicadas al rubro financiero; el objetivo de este estudio fue determinar la relación que existe entre la Gestión del riesgo crediticio con el índice de morosidad en la empresa Mi Banco. El estudio corresponde al enfoque cuantitativo, de tipo no experimental, de nivel descriptivo - correlacional y de corte transversal. Participaron 122 trabajadores de manera voluntaria y corresponde a un tipo de muestreo no probabilístico a quienes se les aplico una escala de riesgo crediticio y otra de índice de morosidad, ambos validados estadísticamente con parámetros aceptables. En base a los resultados se ha encontrado que la correlación Rho de Spearman es Rho $=-0.467$ lo que demuestra que la relación es negativo moderada y el $p=$ es 0,000 menor que 0,05 de manera que se acepta la hipótesis alterna y se rechaza la hipótesis nula, por lo que se infiere que existe relación significativa entre gestión de riesgo crediticio y el índice de morosidad. Finalmente se concluye que existe una correlación directa entre ambas variables estudiadas.

Palabras claves: Gestión; riesgo crediticio; índice; morosidad; banca

\begin{abstract}
In recent times, the management of credit risk and the NPL is a cause of great concern for companies dedicated to the financial sector; The objective of this study is to determine the relationship existing between credit risk management and the NPL rate in the company Mi Banco. The study corresponds to the quantitative approach, non-experimental type, descriptive-correlational level. It was cross-sectional study. 122 workers participated voluntarily corresponding to a type of non-probabilistic sampling to whom a credit risk scale and NPL rate scale were applied, both statistically validated with acceptable parameters.
\end{abstract}

\footnotetext{
* Autor de correspondencia:

E-mail: julio.torres@upeu.edu.pe

DOI: https://doi.org/10.17162/riva.v8i1.1632

Recibido: 8/08/2021 Aceptado: 29/11/2021
} 
Based on the results, it has been found that Spearman's Rho correlation is Rho $=-0.467$, which shows that the relationship is moderately negative and $p=0.000$ less than 0.05 , so that the alternative hypothesis is accepted and the null hypothesis is rejected. It is inferred that there is a significant relationship between credit risk management and NPL rate. Finally, it is concluded that there is a direct correlation between both variables studied.

Keywords: management; credit risk; index; delinquency; banking

\section{Introducción}

Debido a la pandemia del COVID 19 la cantidad de clientes con retraso en el pago se incrementó, los usuarios de las empresas financieras se vieron en dificultades como consecuencia del encierro y la falta de liquidez para hacer frente a las múltiples obligaciones de crédito.

Naranjo (2020) afirma que al inicio de la pandemia el sistema financiero peruano se encontraba con un buen rendimiento, en la actualidad el sistema financiero del país se encuentra en una excelente situación, pero que se tiene que realizar adecuaciones o nuevos programas de pago al sistema de pago de acuerdo con la coyuntura nacional. Esto obedece a que los clientes de las entidades financieras se han visto con problemas por falto de liquidez o en algunos casos imposibilitadas de poder cumplir con sus obligaciones crediticias ante las entidades financieras.

Según el BCRP se prevé que el índice de morosidad en el sistema financiero peruano subiría de 4.6 en febrero a $4.8 \%$ al cierre del año 2020, pero un mayor índice afectaría a los bancos muy puntualmente a los bancos medianos (Solis, 2020).

En los últimos meses se pudo apreciar marchas y múltiples protestas frente a entidades financieras en la región puno ante la imposibilidad de cumplimiento de obligaciones de pago. Según Equifax propietaria de infocorp en su primer informe de morosidad, las reprogramaciones fueron medidas acertadas que ayudo a contener el incremento de morosidad y las regiones que subieron en índice de morosidad son lima callao Arequipa Lambayeque (Soto, 2020).

La entidad financiera mi banco al percibir la incapacidad de pago, otorga a sus clientes la facilidad de posponer sus obligaciones en 30,60, 90 y 180 días de gracia lo que significa el incremento de cuotas a su crédito y reprogramaciones de pago, dependiendo el producto adquirido, también otorgó congelamientos a inicios del estado de emergencia.

Tomando en cuenta que el sistema financiero es muy importante para la economía de un país y de hecho para el nuestro, está también la masificación del número de créditos 
otorgados por las instituciones financieras a sus clientes, sin poner en riesgo la rentabilidad que como toda empresa debe de generar, evitando y utilizando mecanismos para evitar la morosidad, estos procedimientos o protocolos de crédito tienen que ser los acertados, para que los buenos, nuevos y clientes en general siempre reciban buenas ofertas de crédito a bajos intereses y de fácil accesibilidad y en este proceso no se caiga en errores en la gestión crediticia, puesto que hoy por hoy una problema más común de lo que parece, a la que se enfrentan las entidades financieras es la elevada tasa de morosidad, esto puede deberse a la falta de una buena evaluación crediticia.

Una no correcta evaluación puede desencadenar en un riesgo crediticio o los problemas económicos generados actualmente por la pandemia que ha hecho que muchas deudas quedaran impagas por falta de efectivo, es por esta razón que es muy importante realizar nuevos estudios sobre lo que conlleva la gestión de riesgo crediticio y el índice de morosidad, en un contexto no muy normal en la actualidad, si bien es cierto que el riesgo de crédito cero nunca existirá debido a que el universo de las finanzas es extenso, pero se puede evitar, cuando se tenga una excelente y correcta aplicación de gestión de riesgo de crédito que engloba la correcta evaluación del potencial cliente para el otorgamiento del crédito dependiendo el producto solicitado y de modo que esta no sea ni eleve el índice de morosidad que generaría perdida para la entidad crediticia, es por ello la necesidad de la realizar el estudio sobre la relación que existe entre la gestión del riesgo crediticio con el índice de morosidad en mi Banco, agencias de la ciudad de Juliaca y si esto llega a afectar los resultados financieros, económicos y las consecuencias que origina el retraso de la organización en estudio.

En Ecuador, Velasco (2017) realizó un análisis del índice de morosidad y su repercusión en la rentabilidad del sistema de bancos privados grandes situados en la provincia de Imbabura en el tiempo 2014-2016, el objetivo fue analizar el impacto del índice de atrasos en la rentabilidad del sistema. La metodología fue descriptiva. El resultado mostro un $5.15 \%$, pero se ha retomado un crecimiento importante creciendo un $9.73 \%$. Se concluye que la variación del índice de morosidad no implica que la variación en volumen de cartera vencida sea igual, incluso puede suceder que el índice disminuya, sin embargo, el volumen se incrementa.

En Colombia Lara (2010) publicó un artículo sobre el análisis de tendencias en los indicadores financieros de la banca mexicana sobre gestiones de riesgo en instituciones de microfinanzas que tuvo como objetivo sugerir alguna explicación intuitiva de la relación entre el desempeño financiero de los bancos, reflejado en sus razones financieras con el tamaño de los mismos. El resultado del análisis permite conocer acerca del desempeño de los bancos a través de razones o indicadores financieros que miden distintos ámbitos de su actividad, 
además los bancos grandes son los que parecen tener menor motivación por tener suficientes precauciones de cartera vencida, sin embargo, no tienen alta volatilidad indicando así un menor riesgo.

De acuerdo con las tendencias, se puede observar que el sistema bancario en general ha empeorado claramente sus diferentes índices en el periodo de crisis financiera mundial, mostrando variaciones preocupantes que nos advierten de la necesidad de acciones inmediatas para evitar una gran crisis o colapso del sistema bancario mexicano.

En Ecuador, Parrales (2013) realizó una investigación que tuvo como objetivo identificar las primordiales causas por las que se retrasan y promover los sistemas de prevención, el método no experimental descriptiva, correlacional. El resultado fue que la tendencia temporal de los indicadores de morosidad irá en aumento de un $12.54 \%$ y un $12.32 \%$. Concluyendo así que la cartera de créditos no llega a ser aceptable por la morosidad del $10 \%$.

En Trujillo, Gasco (2018) realizó una investigación titulada Gestión del riesgo crediticio y su incidencia en el grado de morosidad de la cooperativa de ahorro y crédito San José de Cartavio de la provincia de Ascope, distrito Santiago de Cao en el periodo 2014-2016 con el objetivo de determinar de qué forma la gestión del riesgo influyo en el grado de morosidad de la Cooperativa. La metodología fue descriptivo correlacional, deductivo. El resultado fue que la morosidad llego un 12.45 \% lo cual da a presentar un grado bajo. Se concluye que se logró identificar los aspectos generales, a través del enfoque COSO/ERM dejando ordenar la estrategia con las metas organizacionales.

En Quillabamba, Yépez (2019) realizó un estudio titulado "Gestión del riesgo crediticio y su influencia en el nivel de morosidad de la Agencia Quillabamba, financiera Credinka S.A. - período 2015" su objetivo fue especificar cómo la gestión del riesgo llega a influir en los niveles de morosidad. El método fue descriptivo, correlacional, no experimental. El resultado fue $62.18 \%$ los cuales respondieron que es regular. Concluyendo que la gestión de riesgo llega a influir considerablemente en la depreciación de la morosidad, lo cual significa una apropiada por parte del analistas o personal de créditos para una intervención proactiva.

En Trujillo, Calderon (2014) realizó una investigación cuyo objetivo fue gestionar el riesgo crediticio y su repercusión en el nivel de morosidad de la caja municipal. El método fue descriptivo-no experimental, inductivo y deductivo. El resultado fue que un $60 \%$ de los entrevistados calificaron como bueno la gestión, por otro lado, el $40 \%$ mencionaron que fue regular. En conclusión, la gestión realizada por la agencia influyo considerablemente bajando la morosidad. 
En Puno, Chambilla (2019) realizó una investigación titulada "Relación de las colocaciones de créditos con la morosidad de los bancos privados de la provincia de Juliaca, San Román año 2016" el objetivo fue saber la opinión de los colaboradores de las entidades financieras privadas sobre el tema de indagación. La metodología fue científica, descriptivo, no experimental. El resultado mostro una contrastación del (8.706), además de una asociación positiva de un $70.2 \%$ de morosidad; del mismo modo de los interesados muestra un $49.28 \%$ de la variación de créditos. Se concluyó que la distribución de créditos se relaciona claramente y significativamente con la demora de cada uno de los clientes en los diferentes bancos.

La gestión de riesgo crediticio según la SBS viene a ser el procedimiento que ayuda a sostener el peligro o amenaza de una financiación o préstamo de acuerdo con las medidas constituidas y planteadas en las políticas y procesos internos para lograr las metas de efectividad y rentabilidad. "Es uno de los primordiales riesgos financieros esto viene a ser la latente falta de cumplimiento originado por la real inviabilidad o repudio, falto de interés de un cliente para asumir sus obligaciones, esa falta de cumplimiento inquieta a los encargados de la gestión de riesgo en el nivel de exposición al peligro de los clientes de la entidad financiera" (Calle, 2018) .

La finalidad principal de la gestión de riesgo crediticio es de proteger la solvencia patrimonial y financiera de un banco en concordancia con sus objetivos estratégicos de expansión y redito. En cuanto al riesgo de crédito comprende o abarca desde la solicitud, monitoreo hasta la recuperación en caso sea pertinente; es decir al total del proceso o periodo de crédito (Cristobal, 2020).

Es la oportunidad de que, al instante del término, una institución no llegue a realizar frente, en su generalidad o parte de ella y a su deber de reembolsar un rendimiento o deuda pactado sobre una herramienta financiera, debido a una liquidez, quiebra, o algún otro motivo, (Alde, 2018). Al respecto, existen factores que determinan el riesgo en instituciones crediticias, estos pueden ser internos, estos dependen de la administración de la entidad financiera y externos, se refieren a la inflación, devaluación de la moneda.

Morosidad se entiende por el retaso de pago del importe pactado por el deudor, cuando una persona, empresa u organización pide un crédito a un banco, hace un compromiso de pago según modalidad, importe y fecha; pero no realiza lo pactado, esto repercute en la renta de la entidad financiera (Bobadilla, 2019). Además, se entiende por índice de morosidad al porcentaje de endeudamiento que tienen las instituciones bancarias o crediticias del incumplimiento de obligaciones adquiridas de parte de los clientes. Según Domínguez (2013) afirma que "llega a medir la magnitud de créditos considerados morosos sobre el general de programación de crédito y préstamo otorgado por una institución financiera". 
Teniendo en cuenta la información que se posee del historial de pago, liquidez capacidad de pago, las deudas que tiene, se reconoce el cliente moroso que se trata. Los especialistas en el tema aconsejan que la negociación en forma directa con el deudor es una buena solución, tomando la alternativa de fijar un nuevo plazo o fraccionar (Brachfield, Brachfiel Credit \& Risk Consultants, 2014). Según Thelma (2016) cartera morosa "son aquellos clientes que, por algún motivo, causa tienden a presentar algunos retrasos en sus cuotas de pago correspondiente". Al respecto, Ramírez (2016) sostiene que la cartera vencida es "la parte del total de sus deudores los cuales se reportan como demora en el crédito de sus préstamos, en otras palabras, la componen los clientes que por algún motivo no pagan, no pueden pagar o que ya están en riesgo judicial."

\section{Materiales y Métodos}

\subsection{Diseño}

Pertenece a un enfoque cuantitativo, de tipo no experimental, de nivel descriptivo Correlacional y transversal, considerando que los datos se han obtenido en un solo momento y tiempo único (Hernandez, 2019)

\subsection{Participantes}

Se ha considerado un tipo de muestreo no probabilístico intencional y participaron voluntariamente 122 colaboradores, quienes se desenvuelven como asesores crediticios de la Microfinanciera Mi banco del departamento de Puno, así mismo se ha considerado un criterio de inclusión como pertenecer al área de créditos y criterio de exclusión a asesores que no sean parte de la agencia, además a personal que trabaja en la plataforma de las agencias.

\subsection{Instrumentos}

Gestión de Riesgo Crediticio: Propuesto y elaborado por Cóndor Martínez klenner y Taipe Sobrevilla Jhonthan en el 2018, Este cuestionario Gestión de Riesgo Crediticio, mide la capacidad para hacer frente al desarrollo de análisis de riesgo tiene como finalidad un diagnóstico del riesgo de crédito, las políticas, los tipos de crédito, consta de 3 dimensiones y 16 ítems, con una escala tipo Likert con 5 respuestas donde: $1=$ Nunca, $2=$ Casi Nunca, $3=$ A veces, $4=$ casi siempre y 5 = Siempre, la primera dimensión contiene 6 ítems, la segunda dimensión políticas de crédito consta de 6 ítems y la tercera dimensión contiene 4 ítems. Además, que tiene un indice Alfa de Cronbach de 0.807

Índice de morosidad: cuestionario propuesto por Cóndor Martínez klenner y Taipe Sobrevilla Jhonthan en el 2018, es un instrumento breve mide la magnitud de créditos considerados morosos sobre el general de programación de crédito y préstamo otorgado por 
una institución financiera, consta de una dimensión con 16 ítems, con una escala tipo Likert con 4 respuestas donde: 1 = Siempre, 2 = Casi siempre, 3 = Solo a veces, 4 = Nunca. Estos dos instrumentos fueron validados, por dos expertos, referente a las propiedades psicométricas la validez de contenido interna se realizó por medio del juicio de 2 expertos ( $V$ de aiken =99), además la fiabilidad del instrumento Alfa de Cronbach es de un 0.81.

\subsection{Procedimiento y análisis de datos}

Para la aplicación construyó los dos inventarios utilizando la herramienta de GOOGLE forms, para la recopilación de datos se remitió los cuestionarios a los participantes asesores crediticios de la institución financiera participante de esta investigacion, mediante sus correos ó whatsapp. Los asesores que componen la agencia Juliaca están distribuidos por agencias de la siguiente manera: Carabaya Macusani nueve (9), Ayaviri melgar seis (6), Pedro Vilcapaza san Román Juliaca veinte (20), Raúl porras San Román Juliaca veintinueve (29), Túpac Amaru San Román Juliaca veintitrés (23) y Túpac Amaru Matriz San Román Juliaca treinticinco (35). La información se recabó desde el mes de febrero del 2021 hasta agosto del 2021.

Una vez obtenida la información o datos en exel de cada instrumento, se realizó un ordenamiento y selección analizada y tabulada, posteriormente se importaron los datos al Programa Estadístico SPSS, versión 25, una vez procesada, se realizó la presentación en tablas de los resultados con sus respectivos resultados para su análisis.

Se realizó el cálculo estadístico descriptivo sociodemográfico, luego la prueba de normalidad de kolmogorov esmirnov para la determinación del coeficiente de correlación a usar, La finalidad de la prueba de normalidad, es analizar si los datos tienen o no una distribución normal, el test de shapiro wilk se emplea en muestras menores de 50 y kolmogorov esmirnov en mayores de 50 muestras. (Amat,2016). Esta prueba de normalidad nos permite hacer la inferencia de usar el coeficiente de correlación Rho de spearman y finalmente se realiza los análisis de correlación entre las variables de estudio.

\subsection{Aspectos éticos}

Respecto al aspecto ético, en el estudio se ha considerado el consentimiento informado detallado al inicio de los instrumentos usados lo que indica participación voluntaria y confidencialidad de los datos obtenidos, por otro lado, se obtuvo el permiso correspondiente de la alta dirección de la empresa financiera mi Banco del departamento de Puno. 


\section{Resultados}

\subsection{Análisis descriptivo}

Según la tabla 1, del total de la muestra de 122, predomina el sexo masculino con $(52,5 \%)$ referente a la edad el mayor porcentaje $(54,1 \%)$ se encuentra las edades de 31 a 40 años, $(81,1 \%)$ son solteros, con grado académico predominante de técnico (54,9\%), una antigüedad de 4 a 8 años (64,8\%), en su mayoría son profesionales contadores $(51,6 \%$.).

\section{Tabla 1}

Resultados demográficos de los encuestados

\begin{tabular}{|c|c|c|c|}
\hline Variables & Categorías & Frecuencia & Porcentaje \\
\hline & Masculino & 64 & 52,5 \\
\hline \multirow[t]{5}{*}{ Sexo } & Femenino & 58 & 47,5 \\
\hline & Total & 122 & 100,0 \\
\hline & 18 a 25 años & 6 & 4,9 \\
\hline & 26 a 30 años & 39 & 32,0 \\
\hline & 31 a 40 años & 66 & 54,1 \\
\hline \multirow[t]{4}{*}{ Edad } & 41 a 50 años & 10 & 8,2 \\
\hline & 51 a más años & 1 & ,8 \\
\hline & Total & 122 & 100,00 \\
\hline & Soltero & 99 & 81,1 \\
\hline Estado & Casado & 22 & 18,1 \\
\hline \multirow[t]{3}{*}{ Civil } & Viudo & 1 & ,8 \\
\hline & Total & 122 & 100,0 \\
\hline & Técnico & 67 & 54,9 \\
\hline Grado & Bachiller & 51 & 41,8 \\
\hline \multirow[t]{4}{*}{ Académico } & Magister & 4 & 3,3 \\
\hline & Total & 122 & 100,0 \\
\hline & 1 a 3 años & 31 & 25,4 \\
\hline & 4 a 8 años & 79 & 64,8 \\
\hline \multirow[t]{5}{*}{ Antigüedad } & 9 a 12 años & 11 & 9,8 \\
\hline & 19 a 24 años & 1 & ,8 \\
\hline & Total & 122 & 100,0 \\
\hline & Contador & 63 & 51,6 \\
\hline & Administrador & 23 & 18,9 \\
\hline \multirow[t]{2}{*}{ Profesión } & Profesor & 7 & 5,7 \\
\hline & Economista & 7 & 5,7 \\
\hline
\end{tabular}




$\begin{array}{ccc}\text { Ingeniero Industrial } & 1 & , 8 \\ \text { Otros } & 21 & 13,3 \\ \text { Total } & 122 & 100,0\end{array}$

Fuente: Elaboración propia

En la tabla 2, nos indica que la prueba de normalidad de kolmogorov-smirnov para la muestra indica que los datos de las variables gestión de riesgo crediticio e índice de morosidad no se distribuyen de manera normal o simétrica, la significancia es de ,000 no es mayor que ,05 en ese sentido el instrumento de correlación a usarse el Rho de Spearman.

\section{Tabla 2}

Prueba de Kolmogorov-Smirnov para muestra

\begin{tabular}{lccc}
\hline & & $\begin{array}{c}\text { Gestión de } \\
\text { riesgo } \\
\text { crediticio }\end{array}$ & $\begin{array}{c}\text { Índice de } \\
\text { morosidad }\end{array}$ \\
\hline $\mathrm{N}$ & Media & 61,61 & 122 \\
Parámetros normales & Desviación Estándar & 7,385 & 28,44 \\
Máximas Diferencias & Absoluta &, 217 &, 216 \\
extremas & Positivo &, 088 &, 216 \\
& Negativo &,- 217 &,- 099 \\
Estadístico de prueba & &, 217 &, 216 \\
Sig. asintótica (bilateral) & &, $000^{c}$ &, $000^{c}$ \\
\hline
\end{tabular}

Fuente: Elaboración propia

\subsection{Análisis de correlación}

En la tabla 3 se distingue los resultados de la correlación entre gestión de riesgo crediticio y el índice de morosidad fueron moderadas con un valor de $\mathrm{Rho}=0.467$ negativo, lo que nos refiere que existe una relación de $46,7 \%$, comprobando la hipótesis, el $p=.0,000$ siendo menor que 0,05 , por lo tanto, se acepta la hipótesis alterna y se rechaza la hipótesis nula.

\section{Tabla 3}

Correlación entre Gestión de riesgo crediticio e índice de Morosidad 


\begin{tabular}{|c|c|c|c|c|}
\hline & & & $\begin{array}{l}\text { Gestión } \\
\text { de riesgo } \\
\text { crediticio }\end{array}$ & $\begin{array}{l}\text { Índice de } \\
\text { Morosidad }\end{array}$ \\
\hline \multirow{6}{*}{ Rho de Spearman } & \multirow[t]{3}{*}{$\begin{array}{l}\text { Gestión de riesgo } \\
\text { crediticio }\end{array}$} & $\begin{array}{l}\text { Coeficiente de } \\
\text { correlación }\end{array}$ & 1,000 &,$- 467^{\star \star}$ \\
\hline & & Sig. (bilateral) & & ,000 \\
\hline & & $\mathrm{N}$ & 122 & 122 \\
\hline & \multirow[t]{3}{*}{ Índice de Morosidad } & $\begin{array}{l}\text { Coeficiente de } \\
\text { correlación }\end{array}$ &,$- 467^{\star *}$ & 1,000 \\
\hline & & Sig. (bilateral) &, 000 & \\
\hline & & $\mathrm{N}$ & 122 & 122 \\
\hline
\end{tabular}

**. La correlación es significativa en el nivel 0,01 (2 colas).

En la tabla 4 el estudio indica que la relación entre evaluación de riesgo crediticio con índice de morosidad es una correlación baja de $\mathrm{Rho}=0.221$, lo cual indica que existe relación negativa del $22,1 \%$; con un $p=.0,014$ siendo menor que 0,05 ; esto indica que se acepta la hipótesis alterna; se concluye, la evaluación de riesgo crediticio se relaciona negativamente con el índice de morosidad en mi banco, agencias de la ciudad de Juliaca.

\section{Tabla 4}

Correlación entre Evaluación de riesgo crediticio e índice de Morosidad

\begin{tabular}{|c|c|c|c|c|}
\hline & & & $\begin{array}{l}\text { Evaluación } \\
\text { de riesgo } \\
\text { crediticio }\end{array}$ & $\begin{array}{l}\text { Índice de } \\
\text { Morosidad }\end{array}$ \\
\hline \multirow{6}{*}{$\begin{array}{c}\text { Rho de } \\
\text { Spearman }\end{array}$} & $\begin{array}{l}\text { Evaluación de } \\
\text { riesgo crediticio }\end{array}$ & $\begin{array}{c}\text { Coeficiente de } \\
\text { correlación }\end{array}$ & \multirow[t]{2}{*}{1,000} &,$- 221^{\star \star}$ \\
\hline & & Sig. (bilateral) & & ,014 \\
\hline & & $\mathrm{N}$ & 122 & 122 \\
\hline & \multirow[t]{3}{*}{$\begin{array}{l}\text { Índice de } \\
\text { Morosidad }\end{array}$} & $\begin{array}{c}\text { Coeficiente de } \\
\text { correlación }\end{array}$ &,$- 221^{\star *}$ & \multirow[t]{2}{*}{1,000} \\
\hline & & Sig. (bilateral) & ,014 & \\
\hline & & $\mathrm{N}$ & 122 & 122 \\
\hline
\end{tabular}

**. La correlación es significativa en el nivel 0,01 ( 2 colas).

En la tabla 5, nos muestra la relación entre políticas de crédito con índice de morosidad, tabla 5 , en el estudio se obtuvo una correlación Rho= -,435 lo cual indica que existe relación negativa moderada del 43.5\%; contrastando la hipótesis aquí también se tiene 
que el $p=.0,000$ es menor que 0,05; por lo que se acepta la hipótesis alterna; concluyéndose que la política de crédito se relaciona significativamente y en forma negativa con el índice de morosidad en mi banco, agencia de la ciudad de Juliaca.

\section{Tabla 5}

Correlación entre políticas de crédito e índice de Morosidad

\begin{tabular}{|c|c|c|c|c|}
\hline & & & $\begin{array}{l}\text { Políticas } \\
\text { de crédito }\end{array}$ & $\begin{array}{l}\text { Índice de } \\
\text { Morosidad }\end{array}$ \\
\hline \multirow{6}{*}{$\begin{array}{c}\text { Rho de } \\
\text { Spearman }\end{array}$} & Políticas de crédito & Coeficiente de correlación & 1,000 &,$- 435^{\star *}$ \\
\hline & & Sig. (bilateral) & &, 000 \\
\hline & & $\mathrm{N}$ & 122 & 122 \\
\hline & $\begin{array}{l}\text { Índice de } \\
\text { Morosidad }\end{array}$ & Coeficiente de correlación &,$- 435^{\star *}$ & 1,000 \\
\hline & & Sig. (bilateral) & ,000 & \\
\hline & & $\mathrm{N}$ & 122 & 122 \\
\hline
\end{tabular}

${ }^{* *}$. La correlación es significativa en el nivel 0,01 (2 colas).

La tabla 6 indica el valor estadístico entre tipos de crédito y el índice de morosidad, es Rho= -0.163 , por lo que se puede sostener que existe relación muy baja del $-16.3 \%$; contrastando la hipótesis se tiene que el $p=., 028$ es menor que 0,05 ; lo cual indica que se acepta la hipótesis alterna; por lo tanto, se concluye que los tipos de crédito se relacionan negativamente con el índice de morosidad.

Tabla 6

Correlación entre tipos de crédito e índice de Morosidad

\begin{tabular}{|c|c|c|c|c|}
\hline & & & $\begin{array}{l}\text { tipos de } \\
\text { crédito }\end{array}$ & $\begin{array}{l}\text { Índice de } \\
\text { Morosidad }\end{array}$ \\
\hline \multirow{3}{*}{\multicolumn{2}{|c|}{ tipos de crédito }} & Coeficiente de correlación & 1,000 &,- 163 \\
\hline & & Sig. (bilateral) & & ,028 \\
\hline & & $\mathrm{N}$ & 122 & 122 \\
\hline \multirow[t]{3}{*}{ Spearman } & Índice de Morosidad & Coeficiente de correlación &,- 163 & 1,000 \\
\hline & & Sig. (bilateral) & ,028 & \\
\hline & & $\mathrm{N}$ & 122 & 122 \\
\hline
\end{tabular}

Fuente: Elaboración propia 


\section{Discusión}

Los resultados obtenidos en este estudio entre gestión de riesgo crediticio y el índice de morosidad indican que existe una relación moderada negativa es decir que la gestión de riesgo influye en el índice de morosidad, lo que concuerda con Yepez, (2015), donde refiere que la gestión de riesgo de crédito adecuada influye disminuyendo los niveles de morosidad. De la misma forma Ticse, (2015) no hace más avalar este resultado cuando refiere que una efectiva administración de los riesgos crediticios, minimiza su influencia en la morosidad, además la evaluación el análisis y predicción baja los efectos adversos de los riesgos. referente al tema con el estudio de Cóndor y Taipe (2018) en su investigación sobre la evaluación de riesgo crediticio y la relación con índice de morosidad obtuvieron una correlación positiva débil de 0,392. Ulloa (2020) Un alto índice de morosidad requiere reestructuración de las políticas crediticias u cobranzas. Queda claramente evidenciado que a mayor evaluación del riesgo crediticio disminuirá el índice de morosidad, así lo confirma Cabrera (2020) en el estudio realizado a 23 trabajadores se demostró que el $57 \%$ de colaboradores nunca revisan el historial de sus clientes, causando la falta de cumplimiento o morosidad, lo que nos lleva a ver que la evaluación del riesgo crediticio influye de sobre manera con el índice de morosidad. Es muy importante resaltar que los estudios evidencian una concordancia con los resultados del estudio, donde la gestión de riesgo crediticio tiene una implicancia directa inversa en el índice de morosidad

La evaluación de riesgo crediticio y la relación con índice de morosidad aunque baja tiene una relación negativa de 221 entre estas variables, Coincide con Pérez (2017) en su estudio determinó una correlación negativa de ,368 entre evaluación crediticia y riesgo crediticio de los 272 emprendedores el 6,6 \% no tuvieron una adecuada evaluación de crédito y es de suponer que influye en la morosidad, el $21,1 \%$, tuvo elevado nivel de riesgo crediticio demostrando más de 30 días de retraso en sus pagos lo que afecta directamente en la capacidad de pago y repercute en el índice de morosidad. Los resultados de la correlación entre políticas de crédito con índice de morosidad el estudio obtuvo una correlación Rho= ,435 y la correlación entre tipos de crédito y el índice de morosidad, Rho $=-0.163$, existe relación muy baja del $-16.3 \%$; es importante mencionar que existe una similitud referente al tema con el estudio de Cóndor y Taipe (2018) los resultados de la correlación entre tipos de crédito y el índice de morosidad, es Rho= -.524.

\section{Conclusiones}

La investigación determinó la evidencia de la existencia de una relación directa negativa entre la Gestión del riesgo crediticio con el índice de morosidad en Mi Banco, lo que 
significa que en cuanto la gestión de riesgo crediticio sea adecuada y correcta disminuye el índice de morosidad en, pero si es contraria el índice de morosidad aumenta.

Como resultado del análisis se conoce que la evaluación de riesgo crediticio con el índice de morosidad, tiene una relación negativa, baja de $\mathrm{Rho}=0.221$, Cuando se realiza una evaluación de la persona u organización, su experiencia de crédito, rubro de negocio, entre otros se refiere a la evaluación cualitativa por otro lado la evaluación cuantitativa referido a gastos personales, historia de sus créditos fuentes de ingreso y otros además de un aval para que la institución financiera recupere el crédito, se refiere a la evaluación de riesgo crediticio, de esto se infiere que en cuanto no se realice adecuadamente o minuciosamente la evaluación del riesgo crediticio el índice de morosidad ira en aumento, en caso de una minuciosa , adecuada y correcta evaluación de riesgo crediticio bajará el índice de morosidad.

El estudio permitió verificar que la relación entre políticas de crédito con índice de morosidad es una relación negativa moderada del $43.5 \%$. Una acertada política de crédito se basa en un correcto y elevado nivel de la calidad de aplicación de procedimientos, estrategias no solo de aprobación de crédito, también seguimiento y recuperación, protocolos seguidos adecuadamente, si estas son aplicadas correctamente menor será el índice de morosidad es decir será baja, de forma contraria el índice de morosidad será alta.

Se identificó que la relación entre los tipos de crédito y el índice de morosidad es Rho= -0.163 , existiendo una relación muy baja del $-16.3 \%$. Los tipos de crédito pueden ser a corporativos, grandes, medianas, pequeñas y microempresas, créditos de consumo, así como hipotecarios, se demuestra que en cuanto se realice mejor el otorgamiento de crédito a las organizaciones y personas el índice de morosidad bajará de manera contraria si se realiza la prestacion del tipo de crédito inadecuado siempre se tendrá un elevado nivel de índice de morosidad.

\section{Referencias}

Alde, E. (2018,18 de agosto). Los 4 tipos de riesgo de crédito. EALDE. https://www.ealde.es/gestion-de-riesgos-decredito/\#: :text=En\%20Gesti\%C3\%B3n\%20de\%20Riesgos\%2C\%20el,iliquidez\%20o\% 20alguna\%20otra\%20raz\%C3\%B3n.

Amat, J. (2016, enero). Análisis de normalidad gráficos y contrastes de hipótesis. Consultado el

https://www.cienciadedatos.net/documentos/8_analisis_normalidad\#: :text=Los\%20an \%C3\%A1lisis\%20de\%20normalidad\%2C\%20tambi\%C3\%A9n,misma\%20media\%20y \%20desviaci\%C3\%B3n\%20t\%C3\%ADpica. 
Bañuelos, S. (2014,10 de julio). Ejemplo de una política de crédito y cobranza. mx. http://t21.com.mx/opinion/arte-cobrar/2014/07/10/ejemplo-politica-credito-cobranza

Bobadilla, E. (219,23 de marzo). Gestión. Obtenido de en el 2018 Aumento de la modalidad crediticia en el peru: https://n9.cl/e0lcx

Brachfield, P. (2009). Gestión del crédito y cobro. Barcelona: Profit Editorial.

Brachfield, P. (2014,23 de diciembre). Brachfiel Credit \& Risk Consultants. Los seis grandes tipos de deudores: https://perebrachfield.com/blog/morosos-y-pufistas/los-seis-grandestipos-de-deudores/

Calderon, R. (2014). La gestión del riesgo crediticio y su influencia en el nivel de morosidad de la caja municipal de ahorro y crédito de Trujillo - agencia sede institucional - periodo 2013. [Universidad Nacional de Trujillo]. http://dspace.unitru.edu.pe/bitstream/handle/UNITRU/959/calderonespinola_rosa.pdf?s equ

Calle, J. P. (28 de febrero de 2018). Riesgo cero. Obtenido de como gestionar el riesgo cero: https://www.riesgoscero.com/blog/como-gestionar-el-riesgo-crediticio

Carrasco, S. (2018). Metodología de la investigación científica. Lima: Editorial San Marcos.

Chambilla, J. (2019). Relación de las colocaciones de créditos con la morosidad de los bancos privados de la provincia de San Román año 2016. [Universidad Andina Néstor Cáceres Velásquez]. http://repositorio.uancv.edu.pe/handle/UANCV/3212

Condor, K., \& Taipe, J. (2019). Gestión del riesgo crediticio y el índice de morosidad en Mi Banco - Agencia Chupaca - 2018. [Universidad Peruana los Andes]. https://hdl.handle.net/20.500.12848/897

Cristóbal, J. M. (2020,17 de 01). Némesis. Obtenido de Gestión de riesgo de crédito: https://nemesisrisk.com/gestion-del-riesgo-de-credito-2/

Domínguez, R. (2013, 12 de agosto). Índice de morosidad. Expansión. https://www.expansion.com/diccionario-economico/indice-de-

morosidad.html\#: :text=En\%20el\%20\%C3\%A1mbito\%20bancario\%2C\%20el,concedid o\%20por\%20una\%20entidad\%20financiera.

Gasco, J., \& Hilario, M. (2018). Gestión del riesgo crediticio y su influencia en el nivel de morosidad de la cooperativa de ahorro y crédito San José de Cartavio del distrito Santiago de Cao de la provincia de Ascope durante el periodo 2014-2016. [Repositorio Universidad Privada Antenor Orrego]. https://repositorio.upao.edu.pe/bitstream/20.500.12759/4938/1/RE_ADMIN_JOSE.GAS CO_MIGUEL.HILARIO_GESTION.RIESGO.CREDITICIO_DATOS.pdf

Hernández S, R. (2019). Metodología de la investigación (1ํed.). México: Editorial Mc Graw Hill. 
Hernández, J. (2019,17 de septiembre). Emprendedor .pe. Obtenido de Las 3 cosas en que se fijan los bancos al otorgar un crédito: https://n9.cl/kg9f

Naranjo, M. (2020,18 de mayo). Confiep Empresarios haciendo País. Obtenido de ASBANC El sistema financiero peruano: Consultado el 20 de julio ,2020. https://www.confiep.org.pe/noticias/asbanc-el-sistema-financiero-peruano-seencuentra-mejor-posicionado-para-enfrentar-esta-pandemia-en-comparacion-con-lascrisis-previas-de-1998-y-2008/

OCDE. (2020, junio). Perspectivas económicas de la OCDE. Obtenido de La economía mundial en la cuerda floja: https://www.oecd.org/perspectivas-economicas/

Parrales, C. (2013). Análisis del índice de morosidad en la cartera de créditos del IECEGuayaquil y propuesta de mecanismos de prevención de morosidad y técnicas eficientes de cobranzas. [Universidad Politécnica Salesiana]. https://dspace.ups.edu.ec/bitstream/123456789/5322/1/UPS-GT000458.pdf

Quispe, J. (2013, 06 de junio). Emprendedor. Obtenido de Tipos de crédito: consultado 18 de junio, 2020https://emprendedor.pe/finanzas/tipos-de-creditos-en-peru/

Ramírez, J. (2016, 16 de julio). Que es la cartera vencida. Conta. Consultado el 02 de abril, 20200btenido de https://www.soyconta.com/que-es-la-carteravencida/\#: :text=Por\%20el\%20contrario\%2C\%20la\%20cartera,por\%20alguna\%20raz \%C3\%B3n\%20no\%20pagan.

Santiago, T. (2014). Fundamentos de economía. Biblioteca Superintendecnai del Mercado de Valores. Obtenido de https://www.smv.gob.pe/Biblioteca/temp/catalogacion/3.pdf

Seijas, M., Búa, M., Sestayo, R., \& López, S. (2017). La evaluación del riesgo crediticio en las instituciones de micro finanzas: estado del arte. Cimpendium, 17. Obtenido de https://webcache.googleusercontent.com/search?q=cache:AKavSoyM9alJ:https://dialn et.unirioja.es/descarga/articulo/6230472.pdf $+\& c d=1 \& \mathrm{hl}=e s-419 \& \mathrm{ct}=\mathrm{clnk} \& \mathrm{gl}=\mathrm{pe}$

Solís, M. (2020,24 de febrero). mercados y regiones. Obtenido de incremento de morosidad bancaria: https://mercadosyregiones.com/2020/02/24/se-preve-incremento-de-lamorosidad-

bancaria/\#: :text=La\%20morosidad\%20bancaria\%20se\%20encuentra,Reserva\%20del \%20Per\%C3\%BA\%20(BCRP).

Soto, s. (2020,08 de 10). Mundo empresarial. Obtenido de Morosidad de personas se mantuvo constante por reprogramaciones: https://www.mundoempresarial.pe/empresas-mundoempresarial/3057-morosidad-constante-reprogramaciones.html

Thelma, L. (2016). Estrategia para la recuperación de la cartera morosa de ENACALMATAGALPA del periodo 2017-2018. https://repositorio.unan.edu.ni/7537/1/17986.pdf 
Ticona, E. (2018). Evaluación del crédito y su incidencia de morosidad de Mi Banco - Banco de la Microempresa S.A. Agencia Juli del periodo 2015. [Universidad Peruana Unión] http://hdl.handle.net/20.500.12840/1518

Trucharte, C. (11 de 2004). Central de riesgo. https://www.sbs.gob.pe/Portals/0/jer/EDIPUB_VOLUMEN2/4TRUCHARTE.pdf

Valderrama, S. (2015). Estadístico Alfa de Cronbach. México: Editorial.Ediciones Texas.

Lara, J. (2010). La gestión de riesgo de crédito en las instituciones de microfinanza. [Universidad de Granada]. https://www.researchgate.net/profile/Juan-LaraRubio/publication/47441016

Velasco, J. (2017). Análisis del índice de morosidad y su impacto en la rentabilidad del sistema de bancos privados grandes ubicados en la provincia de Imbabura en el periodo 20142016

[Universidad Internacional

SEK]. http://repositorio.uisek.edu.ec/handle/123456789/2636

Yépez, Y. (2019). Gestión del riesgo crediticio y su influencia en el nivel de morosidad de la financiera Credinka S.A. - Agencia Quillabamba - Período 2015. Yachay - Revista Científico Cultural, 7(01), 417-423. https://doi.org/10.36881/yachay.v7i01.94 